\title{
PROGRAMA ESCUELA-EMPRESA, ANTECEDENTE DE FORMACIÓN DUAL: CASO UNIVERSIDAD TECNOLÓGICA DEL NORTE DE AGUASCALIENTES, MÉXICO
}

\author{
SCHOOL-ENTERPRISE PROGRAM, BACKGROUND OF DUAL TRAINING: CASE \\ UNIVERSIDAD TECNOLÓGICA DEL NORTE DE AGUASCALIENTES, MEXICO
}

Marcela Rocha López Universidad Tecnológica del Norte de Aguascalientes

Aguascalientes, México ORCID: http://orcid.org/0000-0002-1880-7633 Correo electrónico: marcela.rocha@utna.edu.mx

Luis ERnesto Alemán Macías Universidad Tecnológica del Norte de Aguascalientes Aguascalientes, México ORCID: $h$ ttp://orcid.org/0000-0003-0124-3963 Correo electrónico: luis.aleman@utna.edu.mx

\section{RESUMEN}

La demanda del sector empresarial de profesionistas competentes requiere que las Instituciones de Educación Superior (IES) oferten programas de estudios relevantes y pertinentes que tengan una aplicación inmediata y respondan a las necesidades de este sector. Una de las acciones concretas de la Universidad Tecnológica del Norte de Aguascalientes (UTNA), es el programa escuela-empresa implementado en la carrera de Contaduría, que resalta la necesidad de vincular lo académico y lo laboral, resultando de esta interrelación un estudiante competente capaz de aplicar los contenidos teóricos en un entorno laboral. El objetivo de este estudio fue identificar los elementos de la formación dual y su aplicación en México, para determinar el grado de relación entre esta y el programa escuela-empresa aplicado en la UTNA. Para esto fue necesario describir las actividades que los estudiantes de la carrera de contaduría de la UTNA realizan durante su estancia en un despacho contable y cómo se logran ligar los contenidos académicos a las mismas. Se analizaron las bitácoras de reportes semanales para identificar las actividades más frecuentes y su posible relación con las asignaturas del plan de estudios, así como el incremento por año de los despachos que permiten trabajar con el programa. Los resultados permitieron identificar actividades frecuentes y su relación con los contenidos teóricos y académicos de los planes de estudio.

Palabras clave: Programa; escuela; empresa; formación dual; trabajo.

\begin{abstract}
The industrial sector demands higher education's institutions to offer relevant study programs that can be applied and that can match the needs of this sector. One concrete action of the Universidad Technologica del Norte de Aguascalientes is the program School-Company, which was implemented in Accounting. It highlights the necessity of linking the academic contents to job activities. As a result of this linking, a skilled student is capable of applying the theoretical contents in the job area. The objective of this study was to identify the elements of Dual Education System and its application in Mexico thus determine the level of relation between this system and the UTNA program School-Company. To do this, it was necessary to describe the activities that UTNA accounting students carry out during the program in an accounting firm and how these activities match the academic contents. Students' weekly reports were analyzed in order to identify the most frequent activities and their possible relation with the subjects of the career's curriculum; as well as, the increase per year of the accounting firms, which agree on working in this program. The results showed that the most frequent activities are related to the theoretical and academic contents of the career's curriculum.
\end{abstract}

Keywords: Program; school; company; dual education system; work. 


\section{INTRODUCCIÓN}

El modelo de Educación Dual nace en Alemania a finales de los años sesenta como claro ejemplo de la necesidad de vincular a la universidad con el mundo laboral. El alumno adquiere los conocimientos teóricos y, a través de la conexión con una empresa, aplica estos conocimientos en un contexto real, con el objetivo de ver su relevancia y su pertinencia, y aprender lo que realmente tiene una aplicación inmediata y útil.

En este modelo, el alumno se desempeña como trabajador en una empresa y adquiere una doble certificación, una por parte de la universidad y otra por parte de la empresa. La empresa está comprometida en desarrollar en el estudiante las competencias necesarias y, posteriormente evaluarlas. Además, un tutor asignado lleva a cabo el seguimiento del estudiante y se encarga de registrar y evaluar el avance del educando.

Este sistema de Educación Dual ha tenido un impacto en el mundo laboral, siendo adoptado por países como Croacia y Suiza mostrando resultados favorables con respecto a su aplicación. En el caso de América Latina, países como Colombia o Chile han optado por adaptarlo de acuerdo con su contexto y necesidades.

En México se implementó la Educación Dual mediante la asesoría de la Cámara México-Alemana de comercio e industria, tomando como base la experiencia en el tema del Colegio Nacional de Educación Profesional Técnica, desarrollándose en 2013 el modelo Mexicano de Formación Dual. Los actores que intervinieron son: la Secretaría de Educación Pública, la Subsecretaría de Educación Media Superior y la Confederación Patronal de la República Mexicana, de manera conjunta.

\section{Antecedentes del modelo Dual en México}

Se establece el Acuerdo No. 06/06/15, que regula la formación dual como una opción educativa de tipo medio superior. De los aspectos más relevantes se destaca la relación que debe tener el plan de estudios con el puesto de aprendizaje en la formación dual, la figura del instructor que es la persona de la empresa, capacitada y certificada, responsable de dirigir las actividades de aprendizaje; un plan de rotación de puestos de aprendizaje y el tutor académico que es el docente responsable de acompañar, dar seguimiento y mediar las experiencias de aprendizaje de los estudiantes en el ámbito laboral y escolarizado, así como elaborar en coordinación con el instructor el plan de formación personalizar y evaluar la formación dual. (SEGOB, 2015)

Las Universidades Tecnológicas (UT) fueron creadas en México en 1991 como organismos públicos descentralizados de los gobiernos estatales; su finalidad es ofrecer ca- rreras que respondan a los requerimientos tecnológicos y organizativos de la planta productiva de bienes y servicios, así como la necesidad de formar cuadros profesionales requeridos en proceso de modernización, acordes con los avances científicos y tecnológicos contemporáneos. Su finalidad es la de contribuir al logro de un equilibrio del sistema educativo, con el objeto de ofrecer estudios de nivel post-bachillerato con mayores oportunidades de empleo y con una mayor inversión educativa pública. (Coordinación General de Universidades Tecnológicas, 2006)

Es así que el diseño de sus programas educativos tiene un marcado enfoque de vinculación con el sector laboral, con un modelo de aprendizaje basado en competencias profesionales que contempla el $70 \%$ práctico y el 30\% teórico. Aun cuando la Dirección General de Universidades Tecnológicas establece que deben tener un $70 \%$ de aprendizaje práctico, las estrategias de enseñanza-aprendizaje continúan optando por un aprendizaje más teórico.

La Universidad Tecnológica del Norte de Aguascalientes (UTNA) cuenta con una matrícula que a inicio del ciclo escolar 2018 - 2019 fue de 3050 alumnos; ofrece 15 carreras de TSU y 10 continuidades para adquirir el título de ingeniería. Entre éstas se encuentra la carrera de Contaduría, que se distingue de las que oferta la UTNA, dado que además de trabajar bajo los lineamientos de la Coordinación General de Universidades Tecnológicas (CGUT), apuesta por el programa de Escuela-Empresa.

El Programa se implementa en el año 2002, a partir del quinto cuatrimestre, en el cual se vinculaba las prácticas que los alumnos realizaban en las empresas y/o despachos con la materia de Integradora, en el 2017 se amplía a cuarto cuatrimestre, se relaciona la materia de contribución de personas físicas con el aprendizaje y la práctica que los alumnos logran en contexto con el entorno laboral mediante el programa.

Se considera como referente la investigación realizada por Rocha, Hernández y Salcedo (2016) en esta se comprueba que el Programa Escuela - Empresa es una estrategia de enseñanza aprendizaje que permite el desarrollo de competencias en los alumnos de Contabilidad. Los objetivos planteados por la investigación fueron: evaluar la aplicación de los conocimientos adquiridos del primero al cuarto cuatrimestre de los alumnos de la carrera de Contaduría, en contexto con el entorno laboral actual, determinar el grado de identificación de las buenas prácticas profesionales desde la perspectiva del alumno e identificar la pertinencia de este programa desde el punto de vista de las empresas participantes y por ultimo generar las recomendaciones prácticas para la réplica del proyecto en otras carreras del área económico administrativo. Los sujetos de esta investigación fueron 62 estudiantes que cursaron el programa en 2016 y 30 empresas. 
A través de esta investigación, los resultados permitieron afirmar que el programa escuela - empresa es una estrategia efectiva de vinculación al incrementar, considerablemente, el número de participantes por parte del sector empresarial y el número de proyectos generados entre el 2015 y 2016, además, aumenta las horas prácticas marcadas por el programa educativo y fortalece el desarrollo del alumno en las competencias de ser y saber hacer.

En el Estado de Aguascalientes, se busca fortalecer a las instituciones educativas integrando en sus programas la formación Dual en algunas de sus carreras, por lo que el Gobierno firmó convenio con empresas para fortalecer el vínculo con la formación académica superior.

Es así que la Universidad Tecnológica del Norte de Aguascalientes plantea la posibilidad de implementar una formación Dual en la carrera de Contabilidad, a partir de ello, se realiza un análisis descriptivo de la estructura del programa escuela - empresa y las aportaciones que se han logrado a través de su implementación.

Es importante identificar que, a partir de la experiencia del Programa Escuela - Empresa, la Universidad cuenta con una base que puede darle un mayor fortalecimiento a la implementación de la formación dual.

Pinos (2014) afirma que:

Los procesos de enseñanza aprendizaje de Contabilidad deben lograr de manera efectiva el desarrollo de competencias contables, el papel del docente que imparte conocimientos de contabilidad es esencial punto de partida debiendo tener claro que el estudiante es el centro de su labor, debe revisar su accionar en el proceso de enseñanza aprendizaje seleccionando las estrategias didácticas apropiadas, listar las competencias a desarrollar en cada curso que sean base para otros niveles de aprendizaje y para el futuro profesional. (p.22)

Competencias profesionales y sus desafíos.

Las competencias son el conjunto de conocimientos, habilidades, intelectuales, valores, actitudes, destrezas y sensibilidades armónicamente integrados que se requieren para cumplir con excelencia en tareas cotidianas que se deben realizar en distintos contextos específicos y de niveles de complejidad. (Espíndola, 2011)

Dextre (2011) asegura que un plan de estudios basado en competencias específicas y generales, para que el plan de estudios sea eficaz, requiere dar mayor importancia a las técnicas de enseñanza-aprendizaje-evaluación que permitan una formación integral basada en el aprendizaje.
Vinculación entre las instituciones educativas y el sector laboral (escuela - empresa)

La Asociación Nacional de Universidades e Instituciones de Educación Superior señala que la vinculación con el entorno establece la necesidad de aplicar mecanismos flexibles que reconozcan y analicen los objetivos, los requerimientos y los tiempos de operación de los sectores social, productivo y de servicios, pero en el diseño de dichos mecanismos urge, ante todo, entender las dinámicas institucionales mediante las cuales son desarrolladas estas actividades. (Asociación Nacional de Universidades e Instituciones de Educación Superior, 2012)

Sayago y Chacón (2006) señalan que las prácticas profesionales permiten comunicar al sujeto practicante con acciones institucionales dentro y fuera del ámbito universitario, por lo que al ser producidas en distintos escenarios los estudiantes logran observar, reflexionar, reconstruir y valorar realidades en su contexto; lo cual influye en las concepciones de enseñanza, aprendizaje y sus modelos facilitando el aprendizaje significativo.

De acuerdo con los enfoques, es claro que el camino al aprendizaje significativo se generará de manera exitosa, si el docente se convierte en facilitador del conocimiento a través de estrategias de aprendizaje pertinentes que vinculen al estudiante con el entorno, primero en un ambiente diseñado, tal como el aprendizaje basado en proyectos que permitan evaluar el nivel de competencia del mismo $y$, posteriormente, mediante la práctica formal que acorte la brecha entre la preparación inicial y la formación en la empresa; de esta manera, aplicar el conocimiento en la solución de problemas y la mejora de su entorno.

Análisis descriptivo de la relación existente entre el programa escuela empresa de la carrera de Contaduría y la formación Dual.

A partir del 2002 al 2019, el Programa Escuela Empresa de la carrera de Contabilidad ha logrado vincular a 580 alumnos anticipadamente en los sectores productivos y sociales, tomando como base la implementación de conocimientos tanto teóricos como prácticos, consolidando así la formación mediante el fortalecimiento de las habilidades y actitudes requeridas para el cumplimiento de su perfil de egreso (Rocha et al, 2016).

\section{Elementos de formación Dual}

Cooperación estrecha entre la institución educativa y el sector laboral y los actores sociales; búsqueda de calidad en la educación en la cualificación que implica la participación en los procesos de la empresa idóneas y que la formación impartida se adapte a las necesidades empresariales, donde tutores cualificados profesional y peda- 
gógicamente, que acompañen al alumno durante todo el proceso para lograr objetivos.

\section{El Programa Escuela - Empresa}

Como parte de la dinámica de la Institución se vincula a los alumnos con las empresas y/o despachos contables, permitiendo establecer puentes y rutas de interacción entre la comunidad académica y los diferentes actores sociales, buscando propiciar conocimientos y aprendizaje teórico práctico, reforzar el desarrollo profesional de los alumnos de la carrera de Contabilidad.

Yoguel (2000) hace referencia al desarrollo de conocimientos técnicos en la empresa y en la posibilidad de que ésta se apropie de conocimiento codificado, considerando que de forma tácita influye de manera considerable en el perfil particular de competencia laboral de los recursos humanos, teniendo como consecuencia que el desarrollo de los procesos de aprendizaje al interior de los sistemas de empresas, además de introducir cambios en las actividades de capacitación requeridas así como en el contenido de los contratos e incentivos generados por el aprendizaje conjunto.

\section{El rol de la UTNA en el Programa Escuela - Empresa}

Vincular al alumno con las empresas y/o despachos contables; supervisar que tareas realizadas tengan relación con su aprendizaje; facilitar la inserción laboral de los alumnos egresados; revisar y actualizar el plan de estudio de la Carrera de Contabilidad.

\section{Ventajas para la empresa y/o despacho del Programa Escuela-Empresa}

Desarrollan mayor capacidad para transferir teoría-práctica; presentan alto grado de lealtad con la empresa formadora; aprecian con mayor claridad sus perspectivas profesionales.

\section{Ventajas para los estudiantes del Programa Escuela-Empresa}

Práctica de su enseñanza teórica y aprendizaje en menor tiempo; formación por competencias y trabajo en equipo; preparación para etapa de estadía en la empresa, así como mayor oportunidad de empleabilidad.

Las experiencias de la vinculación del Programa Escuela Empresa

Durante los 17 años de implementación de la estrategia académica de vinculación denominada Programa Escuela Empresa, se ha buscado establecer lineamientos de operación que formalicen sus actividades, por lo que se eligió una materia curricular que, ligada al programa, permitiera dar seguimiento al mismo y evaluar mediante un proyecto el desempeño del alumno y los conocimientos adquiridos.
Para verificar las actividades que los alumnos realizan en las empresas y/o despachos, se implementó un reporte mediante un formato que registra la cantidad de actividades realizadas durante el día, su descripción y la utilidad en el proceso contable o fiscal según sea el caso, así como la relación de estas con alguna de las materias cursadas.

Dentro de las actividades más destacadas realizadas según el reporte de alumnos de 2019, destacan las siguientes: generación de Registro Federal de Contribuyentes, cambio de régimen tributario, cambio de domicilio fiscal de los contribuyentes, emisión de facturas, declaraciones de información a terceros, declaraciones de información a terceros: declaraciones parciales y anuales de Impuesto sobre la renta (ISR), conciliaciones bancarias, registro contable mediante CONTPAQ, así como archivo de documentos, entre otros. Para realizar la mayoría de las actividades requieren del manejo de la plataforma digital de Servicio de Administración Tributaria.

El reporte es firmado por el responsable de asignación de actividades en el despacho y por el alumno, el mismo que se entrega a la coordinación del programa para su seguimiento.

\section{Formas en que se establece la colaboración}

El Programa Escuela - Empresa consta de la asistencia a la empresa o despacho contable que el alumno realiza en el cuarto y quinto cuatrimestre de la carrera de Contaduría, con una duración de 120 horas distribuidas en 5 horas a la semana en la empresa y que ligará de manera permanente con las materias de: Contribución de personas físicas e Integradora II. Por ello los docentes responsables de dichas asignaturas que le otorgan un porcentaje de la calificación total.

El Programa Escuela - Empresa es considerado como una actividad académica y curricular, por lo que la vinculación de las empresas y despachos estará a cargo de la Dirección de Administración y Contaduría.

El compromiso del alumno con la empresa y la institución educativa se establece mediante una carta compromiso de la responsabilidad, refiriendo la conducta deseada con la que debe conducirse, a fin de mantener la vinculación.

Este estudio es de tipo descriptivo, busca analizar las características del Programa Escuela-Empresa que la UTNA implementa en la carrera de Contaduría, con la finalidad de identificar los aspectos semejantes entre el programa y la formación Dual en México, considerando las actividades que los estudiantes realizan en el proceso de vinculación con la empresa y de igual manera el impacto y ventajas del programa en el aprendizaje de los alumnos. 
La muestra con la que se trabajó fue de 271 alumnos que corresponden a los periodos 2015 al 2019. Se analizaron bitácoras representativas de trabajo de los alumnos y se identificaron las actividades más comunes que se realizan como práctica en el despacho contable durante la duración del programa.

Se estableció una relación de las actividades con los contenidos teóricos y las asignaturas, para verificar que el Programa Escuela-Empresa tenga un impacto de aplicación de conocimientos en un contexto real y útil. Se contabilizó el número de horas y se comparó con lo establecido por las UT. Se identificó el número de despachos contables que han permitido una conexión con la institución para la realización del programa.

\section{CONCLUSIONES}

En relación a la descripción de características tanto del Programa Escuela- Empresa como del modelo de formación Dual, se destaca que su objetivo es semejante, sin embargo, los lineamientos de funcionabilidad cambian, por lo que la investigación arroja que la universidad logra triangular a los participantes del programa. Una mayoría significativa de alumnos continúan su etapa de estadía en las empresas que fueron asignadas, de igual forma se ha identificado el fortalecimiento del alumno en su desarrollo personal y profesional ha logrado con ello el alcance de competencias necesarias para el cumplimiento con el perfil de egreso marcado en el programa educativo para ser Técnico Superior Universitario en Contaduría.

Las bases de datos del programa indican que en los últimos cinco años se han vinculado mediante el Programa Escuela - Empresa a 271 alumnos de la carrera de Contaduría con un total de 89 empresas, quedando de la siguiente manera: 2015, 40 alumnos y 22 empresas; 2016, 61 alumnos y 47 empresas; 2017, 44 alumnos y 30 empresas; 2018, 64 alumnos y 44 empresas y por últimos en 2019, 62 alumnos y 46 empresas, que a partir del 2016 el programa duplicó el número de horas.

La Universidad Tecnológica del Norte de Aguascalientes aún no cuenta con la implementación del modelo Dual, pero esta sí otorga a los alumnos del programa de Contaduría una estrategia de enseñanza aprendizaje, que permite un aumento de 120 horas de prácticas más que las marcadas en el programa educativo a nivel sistema UT, que la matricula genera aumento en la cantidad de despachos vinculados, la existencia de una coordinación del programa y dos materias curriculares vinculadas al aprendizaje del alumno, así como un colaborador en la empresa.

La implementación del Programa Escuela-Empresa permite a la carrera de Contabilidad que se integre de manera más eficiente la formación Dual, considerando la experiencia acumulada en los últimos 17 años, por lo que logra identificar como áreas de oportunidades: La importancia de contar con tutores cualificados, un convenio determinado por alumno, compromiso efectivo de la empresa, los planes de rotación y por último el fortalecimiento al aprendizaje del alumno en cuanto al nivel de competencias desarrolladas, mediante una evaluación aplicada por la Institución educativa y avalada por la empresa.

El Programa Escuela-Empresa, con 17 años de funcionamiento, considera que uno de los resultados logrados es la orientación de la malla curricular a la práctica en empresa, lo que ha permitido que los estudiantes en Contaduría tengan una inserción temprana en el ámbito empresarial, teniendo a su alcance las bases de práctica contable y conocimiento básico de software utilizados por la mayoría de las empresas, lo que permite establecer un proceso educativo estrechamente ligado a los problemas y necesidades propias de su profesión.

De acuerdo con los datos registrados en los últimos cinco años de la implementación del programa, se puede decir que, este parece ser la base para la aplicación del modelo educativo de formación Dual, y hace proceso algo más riguroso y sistematizado que nos permita hablar de una formación Dual.

\section{REFERENCIAS BIBLIOGRÁFICAS}

Asociación Nacional de Universidades e Instituciones de Educación Superior, (2012). Inclusión con responsabilidad social, Una nueva generación de politicas de educación superior. México D.F.: Asociación Nacional de Universidades e Instituciones de Educación Superior. Obtenido de https://www.ses.unam.mx/curso2012/ pdf/ANUIES_inclusion.pdf

Coordinación General de Universidades Tecnológicas. (2006). Historia de las Universidades Tecnológicas. En CGUT (Ed.), XV años Universidades Tecnológicas México D.F: Diseños e Impresos de Querétaro S.A de C.V.

Dextre, J. (2011). La dimensión humanística en la formación del contador público. Contabilidad y Negocios, 6(11), 49-55.

Espíndola, C. (2011). Reingeniería educativa, enseñar y aprender por competencias. México D.F: Cenage Learning.

Pinos, M. (2014). Las Competencias Claves y su Relación con las competencias contables. Gaceta Contable, 1(4), 21-30.

Rocha, M., Hernández R., \& Salcedo, P. (2016). Programa Escuela-Empresa, estrategia efectiva de en- 
señanza aprendizaje y vinculación. En Arechavala, R., y García, B. (Comps.), Panorama de Investigación de las Ciencias Económico Administrativas. México D.F: International Council for Small Business México, A.C.

Sayago, Z., \& Chacón, M. (2006). Las prácticas profesionales en la formación docente, hacía un nuevo diario de ruta. Educere, 10 (32), 55-66.
SEGOB (2015) Acuerdo No.06/06/15 por el que se establece la formación dual como una opción educativa del tipo medio superior. Diario Oficial Federal. Obtenido de: http://dof.gob.mx/nota_detalle.php?codigo $=5396202 \&$ fecha $=11 / 06 / 2015$.

Yoguel, G. (2000). Creación de competencias en ambientes locales y redes productivas. Revista de la CEPAL, 71, 105-119. 